\title{
不同幅度的实验增温对藏北高寒草甸净生态系统 碳交换的影响
}

\author{
朱军涛 $^{1}$ 陈 宁 ${ }^{1}$ 张扬建 $^{1,2,3^{*}}$ 刘瑶杰 $^{1}$
}

${ }^{1}$ 拉萨高原生态试验站, 生态系统网络观测与模拟重点实验室, 中国科学院地理科学与资源研究所, 北京 $100101 ;{ }^{2}$ 中国科学院青藏高原地球科学卓越 创新中心, 北京 $100101{ }^{3}$ 中国科学院大学, 北京 100049

摘 要 全球气候变暖将对陆地生态系统(尤其是高寒草甸生态系统)碳循环产生深远影响。该研究依托中国科学院地理科学 与资源研究所藏北高原草地生态系统研究站(那曲站), 设置不同增温幅度实验, 模拟未来2 ${ }^{\circ} \mathrm{C}$ 增温和 $4{ }^{\circ} \mathrm{C}$ 增温的情景, 探究 不同增温幅度对青藏高原高寒草甸净生态系统碳交换(NEE)的影响。研究结果显示: 1)在2015年生长季(6-9月), 不增温和2 ${ }^{\circ} \mathrm{C}$ 增温处理下 $N E E$ 小于 0 , 总体表现为碳汇, 而 $4{ }^{\circ} \mathrm{C}$ 增温处理下 $N E E$ 大于 0 , 总体表现为碳源; 2)在生长季的6月、8月及整个生长 季, 与不增温相比, $4{ }^{\circ} \mathrm{C}$ 增温处理显著提高了 $N E E$, 而 $2{ }^{\circ} \mathrm{C}$ 增温处理没有显著改变 NEE; 7 月, $2{ }^{\circ} \mathrm{C}$ 和 $4{ }^{\circ} \mathrm{C}$ 增温处理均显著提高 了 NEE; 3)在半干旱的高寒草甸生态系统, 土壤水分是决定 $N E E$ 的关键因素, 增温通过降低土壤水分而导致高寒草甸生态系 统碳汇能力下降。该研究可为青藏高原高寒草甸生态系统应对未来气候变化提供基础数据和理论依据。

关键词 气候变暖; 高寒草甸; 净生态系统碳交换; 碳收支; 藏北高原

引用格式: 朱军涛, 陈宁, 张扬建, 刘瑶杰 (2016). 不同幅度的实验增温对藏北高寒草甸净生态系统碳交换的影响. 植物生态学报, 40, 1219-1229. doi: $10.17521 /$ cjpe.2016.0186

\section{Effects of experimental warming on net ecosystem $\mathrm{CO}_{2}$ exchange in Northern Xizang alpine meadow}

ZHU Jun-Tao ${ }^{1}$, CHEN Ning ${ }^{1}$, ZHANG Yang-Jian ${ }^{1,2,3^{*}}$, and LIU Yao-Jie ${ }^{1}$

${ }^{1}$ Lhasa Plateau Ecosystem Research Station, Key Laboratory of Ecosystem Network Observation and Modeling, Institute of Geographic Sciences and Natural Resources Research, Chinese Academy of Sciences, Beijing 100101, China; ${ }^{2}$ Center for Excellence in Tibetan Plateau Earth Sciences, Chinese Academy of Sciences, Beijing 100101, China; and ${ }^{3}$ University of Chinese Academy of Sciences, Beijing 100049, China

\section{Abstract}

Aims Global warming could have profound effects on ecosystem carbon (C) fluxes in alpine ecosystems. The aim of our study is to examine the effects of gradient warming on net ecosystem carbon exchange (NEE).

Methods In the Northern Tibetan Grassland Ecosystem Research Station (Nagqu station), Institute of Geographic Sciences and Natural Resources Research, Chinese Academy of Sciences, we conducted various levels of temperature increasing experiments (i.e., $2{ }^{\circ} \mathrm{C}$ and $4{ }^{\circ} \mathrm{C}$ increments). The warming was achieved using open-top chambers (OTCs). In total, there were three levels of temperature treatments (control, $2{ }^{\circ} \mathrm{C}$ and $4{ }^{\circ} \mathrm{C}$ increment), and four replicates for each treatment. The ecosystem NEE was monitored every five days during the growing season in 2015.

Important findings Our findings highlight the importance of soil moisture in mediating the responses of $N E E$ to climatic warming in alpine meadow ecosystem. The $4{ }^{\circ} \mathrm{C}$ warming significantly stimulated NEE, except for July measurements. The $2{ }^{\circ} \mathrm{C}$ warming had no effects on NEE during the growing season. Compared to the $2{ }^{\circ} \mathrm{C}$ warming, the $4{ }^{\circ} \mathrm{C}$ warming significantly stimulated NEE. The results showed that our targeted ecosystem acts as a carbon sink under $2{ }^{\circ} \mathrm{C}$ warming, whereas will act as a net carbon source under $4{ }^{\circ} \mathrm{C}$ warming in the future. This study provides basic data and theoretical basis for evaluating the alpine ecosystem's responses to climate change.

Key words global warming; alpine meadow; net ecosystem carbon exchange; carbon budget; Northern Xizang plateau

Citation: Zhu JT, Chen N, Zhang YJ, Liu YJ (2016). Effects of experimental warming on net ecosystem $\mathrm{CO}_{2}$ exchange in Northern Xizang alpine meadow. Chinese Journal of Plant Ecology, 40, 1219-1229. doi: 10.17521/cjpe.2016.0186 
联合国政府间气候变化专门委员会(IPCC)第4 次评估报告指出, 自1850年以来, 全球地表平均温 度大约升高 $0.76{ }^{\circ} \mathrm{C}$, 并在 21 世纪末升高 $1.8-4.0{ }^{\circ} \mathrm{C}$ (IPCC, 2007), 因此温度升高对陆地生态系统的影 响成为生态学家关注的重要问题(傅伯杰等, 2005)。 高纬度、高海拔地区生态系统对气候变化更为敏感, 从而成为气候变化影响研究的热点区域(Thomas et al., 2004)。青藏高原高寒草甸生态系统是适应高原 隆起与长期低温环境形成的特殊产物, 对增温响应 敏感, 在全球气候和碳收支等方面起重要作用(李 英年等, 2004; 元伟伟等, 2012)。研究表明青藏高原 自19世纪50年代以来温度上升显著, 每10年温度上 升 $0.32{ }^{\circ} \mathrm{C}$, 高于全球大气平均增温速度 (Liu \& Chen, 2000)。

全球气候变暖已被证明能促进生态系统的碳吸 收或排放(Rustad et al., 2001; Melillo et al., 2002; Welker et al., 2004; Wan et al., 2005; Zhou et al., 2007)。虽然温度是影响净生态系统碳交换(NEE)的 重要因素(Oberbauer et al., 2007; Niu et al., 2013), 但 影响NEE的因素众多, 如物种组成、生物多样性、光 合作用及初级生产力等生物要素(Harte et al., 2006; Wan et al., 2007; 元伟伟等, 2012), 土壤温湿度、土壤 矿化速率及氮的有效性等非生物要素(Saleska et al., 1999; Xia et al., 2009; 开伟伟等, 2012)及各类生态系 统的异质性(Peñuelas et al., 2004; Corradi et al., 2005) 的共同作用, 导致不同生态系统的碳收支存在差异。 例如, 在干旱的亚寒带生态系统中, 增温对生态系 统呼吸 $(E R)$ 的促进作用超过生态系统总初级生产力 (GPP), 生态系统表现为碳源(Illeris et al., 2004); 而 在干旱半干旱的温带草原, 生态系统表现为碳汇 (Niu et al., 2008; Xia et al., 2009)。生物、非生物因素, 以及生态系统的异质性导致了生态系统碳收支对未 来增温的响应有很大的不确定性。

青藏高原被称为世界第三极, 是气候变化的敏 感区域, 同时也是全球大气温室气体的启动区(高 晓清, 1994)。目前对青藏高原高寒草甸的研究表明, 在自然条件下, 由于生态环境恶劣, 牧草生长时间 短, 光能利用率低, 导致初级生产力水平低下, 因 此高寒草甸生态系统表现为碳源。青藏高原东缘的 BIOMEBGG模拟研究表明, 在大气增温1.2-1.7 ${ }^{\circ} \mathrm{C}$ 的条件下, GPP增加幅度超过 $E R$ 的增加幅度, 最终 表现为碳汇(元伟伟等, 2012)。进一步的研究表明高
寒矮生嵩草(Kobresia humilis) 草甸或退化草地无论 表现为碳源或碳汇, 均是一个小源或小汇, 因年际 气候变化而有所不同(张金霞等, 2003)。

综上所述, 多数增温对生态系统碳循环影响的 研究只检验增温和不增温的效果, 模拟不同增温幅 度对生态系统碳循环影响的研究较少。此外, 由于 实验增温对生态系统碳循环的影响研究存在诸多分 歧, 为了更好地理解生态系统碳循环的温度敏感性 问题, 亟需通过设置不同增温幅度的实验来探究增 温对生态系统碳循环的影响(Luo, 2007)。本研究依 托中国科学院地理科学与资源研究所藏北高原草地 生态系统研究站(那曲站), 利用开顶箱(OTCs)模拟 未来 $2{ }^{\circ} \mathrm{C}$ 增温和 $4{ }^{\circ} \mathrm{C}$ 增温的情景, 研究不同增温幅 度对藏北高原高寒草甸 NEE 的影响, 旨在探究全球 变暖情景下, 增温对高寒草甸生态系统碳收支的影 响, 为青藏高原高寒草甸生态系统应对未来气候变 化提供基础数据和理论依据。

\section{1 材料和方法}

\section{1 研究区域概况}

实验地位于那曲站围栏内, 2011年10月开始围 栏禁牧。那曲站 $\left(31.64^{\circ} \mathrm{N}, 92.02^{\circ} \mathrm{E}\right.$, 海拔4 $\left.585 \mathrm{~m}\right)$ 隶属于中国科学院地理科学与资源研究所高原生态 系统研究中心, 位于藏北核心地带那曲地区那曲县 那曲镇曲果仁毛村境内。那曲县属于高原亚寒带季 风半湿润气候区。平均海拔4 $500 \mathrm{~m}$ 以上, 高寒缺氧, 气候干燥。全年大风日 100 天左右, 平均风速 2.7 $\mathrm{m} \cdot \mathrm{s}^{-1}$, 最大风速可达 $26.3 \mathrm{~m} \cdot \mathrm{s}^{-1}$; 年平均气温 -1.16 ${ }^{\circ} \mathrm{C}$, 全年日照时间 $2788 \mathrm{~h}$; 年降水量 $430 \mathrm{~mm}$, 降水 集中在6-9月, 占全年降水量的85\% (以上数据来自 中国气象科学数据共享服务网那曲站点)。全年无绝 对无霜期, 每年10月至次年5月为风雪期和土壤冻 结期(朱军涛, 2016)。

该区域植被类型为典型的高寒草甸, 优势种为 高山嵩草(Kobresia pygmaea), 常见伴生种有钉柱委 陵菜(Potentilla saundersiana)、无茎黄鹤菜(Youngia simulatrix)、藏西风毛菊(Saussurea stoliczkai)、矮羊 茅 (Festuca coelestis)、楔叶委陵菜 (Potentilla cuneata)、矮火线草(Leontopodium nanum)等。土壤 类型为高寒草甸土(朱军涛, 2016)。

\section{2 实验设计}

本实验研究对象为自然状态下的高寒草甸生态 
系统, 采用OTCs式增温装置。利用OTCs的高度控 制增温的效果, 设置了 2 个增温幅度。每个OTCs的 顶边长均为 $80 \mathrm{~cm}$, 保证所有处理的OTCs开口大小 一致, 底边长分别为 100 和 $130 \mathrm{~cm}$, 高度分别为 40 和 $100 \mathrm{~cm}$, 其中 $2{ }^{\circ} \mathrm{C}$ 增温和 $4{ }^{\circ} \mathrm{C}$ 增温处理的样方面积 分别为 1.73 和 $4.39 \mathrm{~m}^{2}$, 样方间距 $2.5 \mathrm{~m}$ (附件I)。 OTCs均采用三角钢支撑, 四周材质为透明玻璃纤 维, 透光率达到 $90 \%$ 以上(朱军涛, 2016)。本实验设 置3种处理, 包括不增温、 $2{ }^{\circ} \mathrm{C}$ 增温、 $4{ }^{\circ} \mathrm{C}$ 增温, 其中 每个处理设置 4 个重复, 共计 12 个样方, 增温处理均 为全年增温。OTCs于2013年9月在那曲站完成安装 (朱军涛, 2016)。

\section{$1.3 \quad N E E$ 的测量}

在不同增温实验处理样地中心布设一个基座为 $5 \mathrm{~cm}$ 高的正方形铁框, 并将铁框打入土壤约 $3 \mathrm{~cm}$, 防止气体泄露。在2015年生长季, 选择晴朗天气在 9:00-12:00对增温实验处理的 NEE进行连续测定, 每隔 5 天测定一次。测定时将体积为 $50 \mathrm{~cm} \times 50 \mathrm{~cm} \times$ $50 \mathrm{~cm}$ 的透明交换箱与红外气体分析仪(LI-6400, LI-COR, Lincoln, USA)连接, 并放到实验样方的底 座上, 箱子中部安装两个风扇混匀箱内气体, 待系 统稳定后, 开始记录 $\mathrm{CO}_{2}$ 的浓度变化, 每次测定时 间为 $90 \mathrm{~s}$, 测定间隔为 $10 \mathrm{~s}$ (Niu et al., 2008)。

$N E E$ 通过以下公式计算:

$$
N E E=\frac{30 \times K \times \operatorname{Press} \times((1-y) / 1000)}{8.314 \times\left(273.15+T_{\text {air }}\right)}
$$

公式(1)中的 $K$ 表示透明箱中 $\mathrm{CO}_{2}$ 体积变化的速率, 即在箱体透光状态下, 用 $90 \mathrm{~s}$ 内连续测得并记录的 9 次 $\mathrm{CO}_{2}$ 浓度数值求得的变化斜率, $y$ 表示在测定过程 中透明箱中 $\mathrm{H}_{2} \mathrm{O}$ 的体积, Press是大气压强 $(\mathrm{Pa}), T_{\mathrm{air}}$ 是 箱室内的温度 $\left({ }^{\circ} \mathrm{C}\right)$ 。

\section{4 空气和土壤温湿度测定}

利用HMP155A探头(Vaisala, Helsinki, Finland) 记录地上 $10 \mathrm{~cm}$ 处的空气温湿度, 测量时间间隔为 30 min。利用CS655探头(Campbell Scientific, Logan, USA)记录地下 $5 \mathrm{~cm}$ 的土壤温湿度, 测量时间间隔 为 $30 \mathrm{~min}$ 。每个实验处理安装 2 套空气和土壤温湿度 探头, 小气候数据为 2 套探头的平均值。

\section{5 数据分析}

采用单因素方差分析检验不同增温实验处理间 土壤湿度、土壤温度及空气温度的差异。采用重复 测量方差分析检验生长季及不同时间段增温处理对
$N E E$ 的影响。主体内效应检验测量时间对 $N E E$ 的影 响, 主体间效应检验增温实验处理对 $N E E$ 的影响。 采用Tukey HSD检验增温实验处理间的差异性。上 述统计分析均在SPSS 21.0中实现。

\section{2 结果}

\section{1 实验增温对小气候的影响}

2015年生长季6-9月降水量为 $238 \mathrm{~mm}$ ，低于同 时期(6-9月)多年平均降水量365 mm, 且表现出明 显的季节变异，降水集中于 6 月 $(73.9 \mathrm{~mm})$ 和 8 月 (117.3 mm), 7月降水最低, 仅为 $40.1 \mathrm{~mm}$ (图1)。在整 个植物生长季, 不增温、 $2{ }^{\circ} \mathrm{C}$ 增温和 $4{ }^{\circ} \mathrm{C}$ 增温处理的 土壤湿度均随降水量的变化而改变, 但表现出2-3 天的滞后期(图1)。不增温、 $2{ }^{\circ} \mathrm{C}$ 增温和 $4{ }^{\circ} \mathrm{C}$ 增温处 理的土壤湿度变化趋势一致, 但土壤湿度依次是不 增温 $>2{ }^{\circ} \mathrm{C}$ 增温 $>4{ }^{\circ} \mathrm{C}$ 增温, 彼此间的差距随降水量 的增加而增大(图1)。

单因素方差分析表明, 与不增温相比, $4{ }^{\circ} \mathrm{C}$ 增温 处理显著提高土壤温度 $2.3{ }^{\circ} \mathrm{C}(p<0.05)$, 与 $2{ }^{\circ} \mathrm{C}$ 增 温处理相比，显著提高了 $2.0{ }^{\circ} \mathrm{C}(p<0.05)$ (图2A)。 与不增温相比, $2{ }^{\circ} \mathrm{C}$ 增温和 $4{ }^{\circ} \mathrm{C}$ 增温处理显著降低土 壤湿度 $(p<0.05)$, 分别降低了 $2.6 \%$ 和 $7.1 \%$; 与 $2{ }^{\circ} \mathrm{C}$ 增温处理相比, $4{ }^{\circ} \mathrm{C}$ 增温处理显著降低土壤湿度 $4.5 \%(p<0.05)$ (图2B)。

\subsection{5 年生长季增温对 $N E E$ 的影响}

重复测量方差分析表明，测量时间 $(p<0.01)$ 、 增温处理 $(p<0.0)$ 及测量时间和增温处理的交互作 用 $(p<0.1)$ 均显著影响 NEE (表1)。在植物生长季, 不增温、 $2{ }^{\circ} \mathrm{C}$ 增温和 $4{ }^{\circ} \mathrm{C}$ 增温处理的 $N E E$ 表现出一致 的动态趋势。2015年生长季6月到7月中旬, $N E E$ 整体 呈下降趋势, 7 月初, 不增温和 $2{ }^{\circ} \mathrm{C}$ 增温由碳源转为 碳汇, 7 月末, 达到生长季最低值, 分别为 -0.76 和 $-0.38 \mu \mathrm{mol} \mathrm{CO}_{2} \cdot \mathrm{m}^{-2} \cdot \mathrm{s}^{-1}$ (图3); 7月中旬到8月中旬 $N E E$ 表现出上升趋势, 8月初, 不增温与 $2{ }^{\circ} \mathrm{C}$ 增温处 理下, 高寒草甸生态系统由碳汇转为碳源, 8月中旬, 不增温、 $2{ }^{\circ} \mathrm{C}$ 增温和 $4{ }^{\circ} \mathrm{C}$ 增温处理的 $N E E$ 均达到生长 季最高值, 分别为 $0.35 、 0.45$ 和 $0.69 \mu \mathrm{mol} \mathrm{CO} \mathrm{CO}_{2} \cdot \mathrm{m}^{-2} \cdot \mathrm{s}^{-1}$ (图3); 8月中旬到生长季结束, 不增温、 $2{ }^{\circ} \mathrm{C}$ 增温和 4 ${ }^{\circ} \mathrm{C}$ 增温处理的 $N E E$ 均呈下降趋势, 8 月末不增温与 2 ${ }^{\circ} \mathrm{C}$ 增温处理下, 高寒草甸生态系统由碳源再次转化 为碳汇, 此时 $4{ }^{\circ} \mathrm{C}$ 增温处理的 $N E E$ 达到生长季最低 值, 为 $0.004 \mu \mathrm{mol} \mathrm{CO} \cdot \mathrm{m}^{-2} \cdot \mathrm{s}^{-1}$ (图3)。 


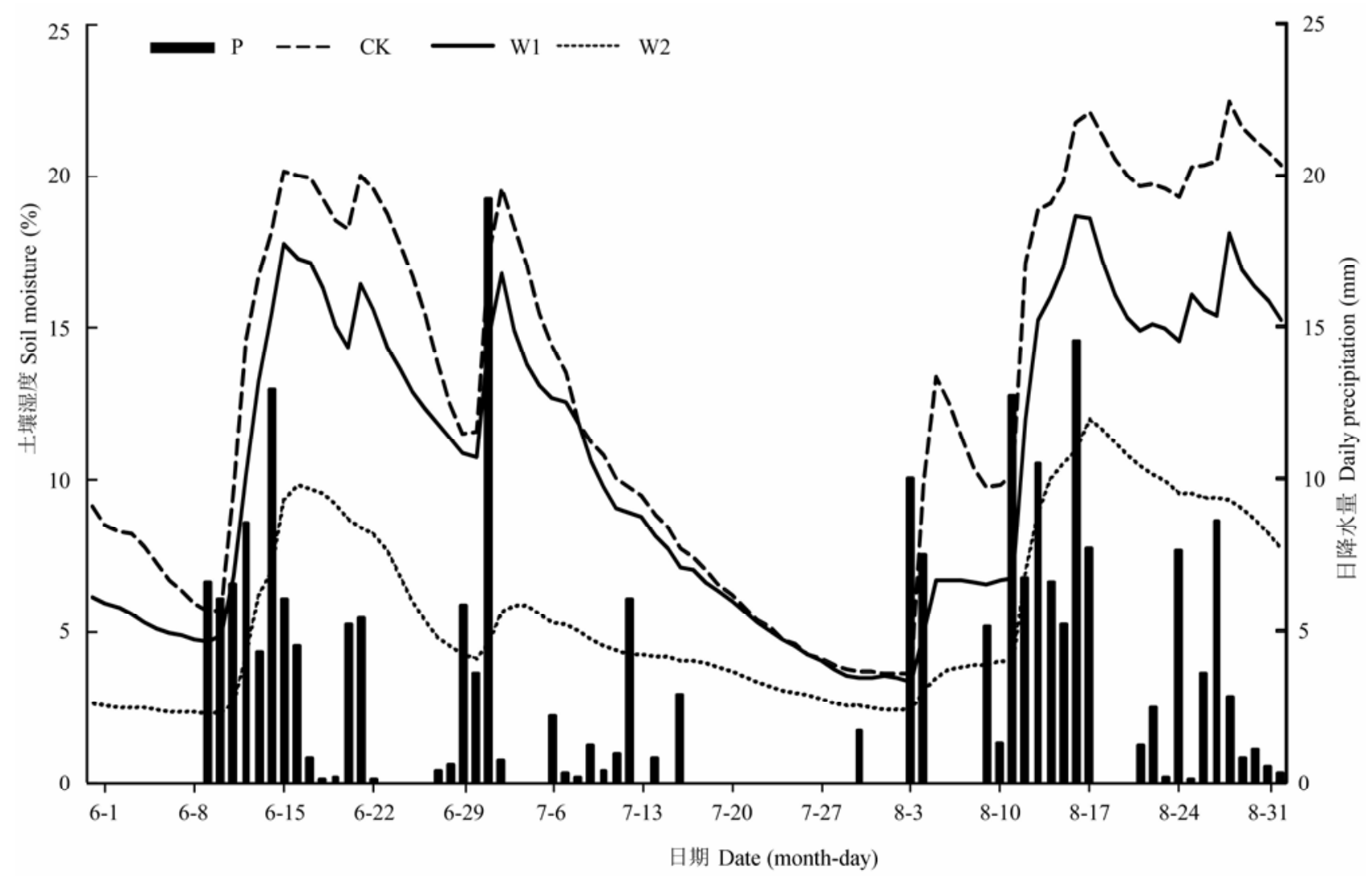

图1 植物生长季日降水量(柱状图)(P)与不同增温幅度的土壤湿度(折线图)。 $\mathrm{CK}$, 不增温; $\mathrm{W} 1 、 \mathrm{~W} 2$ 分别代表模拟未来增温2 ${ }^{\circ} \mathrm{C}$ 和 $4{ }^{\circ} \mathrm{C}$ 的实验处理。

Fig. 1 Daily precipitation (bars) (P) and soil moisture (lines) under different warming treatments during the plant growing season. CK, no warming; W1, W2 represent the simulation of future temperature increase of $2{ }^{\circ} \mathrm{C}$ and $4{ }^{\circ} \mathrm{C}$ experimental treatment.
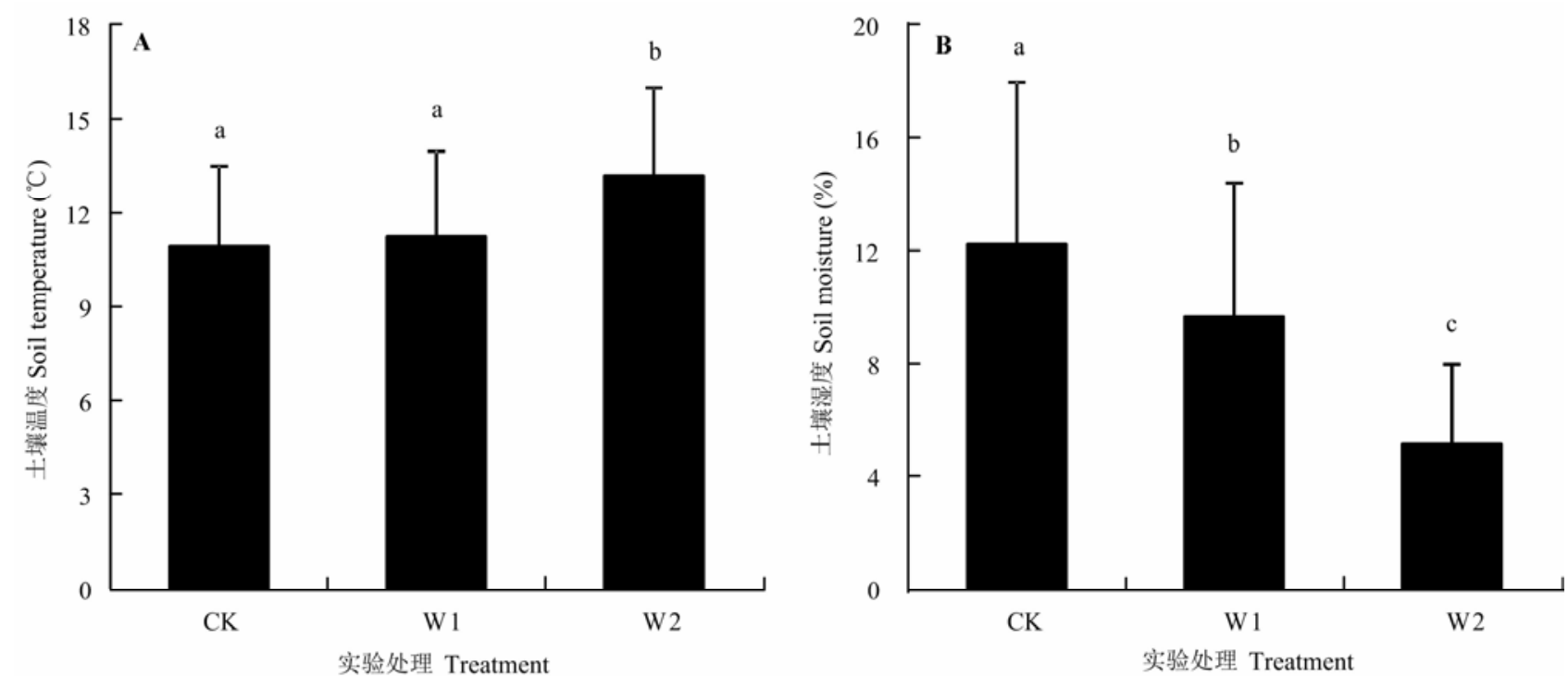

图2 植物生长季不同增温幅度处理下的土壤温度(A)和土壤湿度(B) (平均值土标准误差)。CK, 不增温; W1、W2分别代表模拟 未来增温 $2{ }^{\circ} \mathrm{C}$ 和 $4{ }^{\circ} \mathrm{C}$ 的实验处理。不同小写字母表示在 0.05 水平上差异显著。

Fig. 2 Soil temperature (A), soil moisture (B) during the growing season under different warming treatments (mean $\pm S E$ ). CK, no warming; W1, W2 represent the simulation of future temperature increase $2{ }^{\circ} \mathrm{C}$ and $4{ }^{\circ} \mathrm{C}$. The different lowercase letters at the top of the figures represent significant differences at the 0.05 level. 
表1 增温 $(\mathrm{W})$ 、测量时间 $(\mathrm{T})$ 及其交互作用 $(\mathrm{T} \times \mathrm{W})$ 对净生态系统碳交换 $(N E E)$ 的影响的重复测量方差分析结果

Table 1 Results ( $F$ values) of repeated-measurement ANOVA on the effects of warming $(W)$, measuring date $(T)$, and their interactions $(T \times W)$ on net ecosystem $\mathrm{CO}_{2}$ exchange (NEE)

\begin{tabular}{|c|c|c|c|c|c|c|c|c|}
\hline & \multicolumn{2}{|c|}{ 生长季 Growing season } & \multicolumn{2}{|c|}{ 6月 June } & \multicolumn{2}{|c|}{ 7月 July } & \multicolumn{2}{|c|}{ 8月 August } \\
\hline & $d f$ & $F$ & $d f$ & $F$ & $d f$ & $F$ & $d f$ & $F$ \\
\hline $\mathrm{T}$ & 2.25 & $23.38^{* * *}$ & 2 & $45.78^{* * *}$ & 2.18 & $8.93^{* * *}$ & 5 & $19.22^{* * *}$ \\
\hline W & 2.00 & $8.72^{* *}$ & 2 & $6.58^{* *}$ & 2.00 & $6.58^{* *}$ & 2 & $4.77^{*}$ \\
\hline $\mathrm{T} \times \mathrm{W}$ & 4.51 & 2.27 & 4 & 0.47 & 2.00 & $13.57^{* * * *}$ & 10 & 0.74 \\
\hline
\end{tabular}

*、**、***分别表示在 $90 \% 、 95 \% 、 99 \%$ 的置信水平上具有差异显著性。 $d f$, 自由度。

$*, * *, * * *$ represent statistically significant at the $90 \%, 95 \%, 99 \%$ confidence level. $d f$, degree of freedom.

增温对 $N E E$ 的影响在生长季的不同时期存在显 著差异(图4)。在2015年生长季6月，与不增温相比, 4 ${ }^{\circ} \mathrm{C}$ 增温处理下 $N E E$ 显著提高了 $0.24 \mu \mathrm{mol}$ $\mathrm{CO}_{2} \cdot \mathrm{m}^{-2} \cdot \mathrm{s}^{-1}$, 与 $2{ }^{\circ} \mathrm{C}$ 增温相比, $4{ }^{\circ} \mathrm{C}$ 增温处理下 $N E E$ 显著提高了 $0.10 \mu \mathrm{mol} \mathrm{CO} \mathrm{CO}_{2} \cdot \mathrm{m}^{-2} \cdot \mathrm{s}^{-1}$ (图4A), $2{ }^{\circ} \mathrm{C}$ 增温 与不增温处理之间差异不显著(图4A), 不增温、 ${ }^{\circ} \mathrm{C}$ 增温和 $4{ }^{\circ} \mathrm{C}$ 增温处理下 $N E E$ 均为正值 (图 $4 \mathrm{~A}$ )。在 2015年生长季7月, 实验增温显著提高了 NEE (表1, $p<0.05$ ), 在 $2{ }^{\circ} \mathrm{C}$ 和 $4{ }^{\circ} \mathrm{C}$ 增温处理下, NEE分别提高 了 0.27 和 $0.65 \mu \mathrm{mol} \mathrm{CO}_{2} \cdot \mathrm{m}^{-2} \cdot \mathrm{s}^{-1}$ (图4B), 与 $2{ }^{\circ} \mathrm{C}$ 增温 处理相比, $4{ }^{\circ} \mathrm{C}$ 增温处理下 $N E E$ 显著提高了 0.38 $\mu \mathrm{mol} \mathrm{CO}_{2} \cdot \mathrm{m}^{-2} \cdot \mathrm{s}^{-1}$ (图4B), 除 $4{ }^{\circ} \mathrm{C}$ 增温处理下 $N E E$ 为 正值外, 不增温和 $2{ }^{\circ} \mathrm{C}$ 增温处理下 NEE均为负值(图 4B)。在2015年生长季 8 月, 与不增温相比, $4{ }^{\circ} \mathrm{C}$ 增温 处理下 $N E E$ 显著提高了 $0.42 \mu \mathrm{mol} \mathrm{CO}_{2} \cdot \mathrm{m}^{-2} \cdot \mathrm{s}^{-1}$, 与2 ${ }^{\circ} \mathrm{C}$ 增温处理相比, $4{ }^{\circ} \mathrm{C}$ 增温处理下 $N E E$ 显著提高了 $0.19 \mu \mathrm{mol} \mathrm{CO}_{2} \cdot \mathrm{m}^{-2} \cdot \mathrm{s}^{-1}$ (图4C), $2{ }^{\circ} \mathrm{C}$ 增温处理与不增 温之间差异不显著(图4C), 除不增温处理下 $N E E$ 为 负值外, $2{ }^{\circ} \mathrm{C}$ 增温和 $4{ }^{\circ} \mathrm{C}$ 增温处理下 $N E E$ 均为正值 (图4C)。

在2015年生长季, 与不增温相比, $4{ }^{\circ} \mathrm{C}$ 增温处理 下 $N E E$ 显著提高了 $0.48 \mu \mathrm{mol} \mathrm{CO}_{2} \cdot \mathrm{m}^{-2} \cdot \mathrm{s}^{-1}$, 与 $2{ }^{\circ} \mathrm{C}$ 增 温相比, $4{ }^{\circ} \mathrm{C}$ 增温处理下 $N E E$ 显著提高了 $0.26 \mu \mathrm{mol}$ $\mathrm{CO}_{2} \cdot \mathrm{m}^{-2} \cdot \mathrm{s}^{-1}$ (图4D), $2{ }^{\circ} \mathrm{C}$ 增温与不增温之间差异不 显著(图4D), 不增温与 $2{ }^{\circ} \mathrm{C}$ 增温处理下 $N E E$ 均为负 值, $4{ }^{\circ} \mathrm{C}$ 增温处理下 $N E E$ 为正值(图3A)。与不增温相 比, $4{ }^{\circ} \mathrm{C}$ 增温处理下生态系统呼吸 $(E R)$ 提高了 0.20 $\mu \mathrm{mol} \mathrm{CO} \mathrm{CO}_{2} \cdot \mathrm{m}^{-2} \cdot \mathrm{s}^{-1}$ (图3B, $\left.p>0.05\right)$; 与不增温相比, 4 ${ }^{\circ} \mathrm{C}$ 增温处理下生态系统总初级生产力 $(G P P)$ 提高了 $0.28 \mu \mathrm{mol} \mathrm{CO}_{2} \cdot \mathrm{m}^{-2} \cdot \mathrm{s}^{-1}$ (图3C, $p>0.05$ )。综上分析, 与增温处理 $\left(2{ }^{\circ} \mathrm{C}\right.$ 增温、 $4{ }^{\circ} \mathrm{C}$ 增温)相比, 不增温处理 的碳吸收时间更长、能力更强, 因此增温在一定程 度上会抑制生态系统碳吸收。

\section{$2.3 \quad N E E$ 的影响因素分析}

有关 $N E E$ 与环境因子关系的分析结果表明, 在 2015年生长季, 土壤湿度与生态系统 $N E E$ 呈显著负 相关关系(图5, $p<0.05$ ), 说明土壤含水量越高, 高 寒草甸生态系统固碳能力越强, 但土壤温度与生态 系统NEE没有显著的相关关系(图5, $p>0.1$ ), 说明 对半干旱的高寒草甸生态系统而言, 在干旱的2015 年生长季, 相比于土壤温度, 土壤湿度是决定 $N E E$ 的关键因素。

\section{3 讨论}

\section{1 增温对高寒草甸生态系统碳收支的影响}

对于2015年整个生长季而言, 与不增温相比, 4 ${ }^{\circ} \mathrm{C}$ 增温处理显著提高了 $N E E$; 与 $2{ }^{\circ} \mathrm{C}$ 增温相比, $4{ }^{\circ} \mathrm{C}$ 增温处理同样显著提高了 $N E E$; 不增温和 $2{ }^{\circ} \mathrm{C}$ 增温 处理表现为弱的碳汇, $4{ }^{\circ} \mathrm{C}$ 增温处理则表现为碳源。 在陆地生态系统中, $N E E$ 由 $G P P$ 和 $E R$ 共同决定 (Oberbauer et al., 2007)。BIOMEBGC模型的模拟结 果表明, 在增温1.2-1.7 ${ }^{\circ} \mathrm{C}$ 的条件下, 高寒草甸生态 系统表现为弱的碳汇(元伟伟等, 2012)。我们的研究 表明, 与不增温相比, $2{ }^{\circ} \mathrm{C}$ 增温处理使空气温度升高 了 $1.5{ }^{\circ} \mathrm{C}$, 在整个生长季表现为弱的碳汇, 该结论与 开伟伟等(2012)的研究结果一致。但增温也会降低 土壤水分, 抑制植物的气孔导度、光合作用及 $G P P$ 等(Ciais et al., 2005; Granier et al., 2007; Niu et al., 2008; 郭亚奇等, 2011), 使 $G P P$ 升高幅度低于 $E R$ 提 高幅度。在干旱的亚寒带生态系统中, 增温对 $E R$ 的 提高程度大于 GPP, 导致其表现为碳源(Illeris et al., 2004)。我们的研究表明, 与不增温相比, $4{ }^{\circ} \mathrm{C}$ 增温处 理显著提高土壤温度 $2.3{ }^{\circ} \mathrm{C}$ 和降低土壤湿度 $7.1 \%$, 导致土壤水分显著下降, 进而抑制了 $G P P$ 。与不增 温相比, $4{ }^{\circ} \mathrm{C}$ 增温处理下 $E R$ 增加 $0.20 \mu \mathrm{mol}$ $\mathrm{CO}_{2} \cdot \mathrm{m}^{-2} \cdot \mathrm{s}^{-1}(p>0.05$; 图3B), 而 $G P P$ 增加 $0.28 \mu \mathrm{mol}$ 

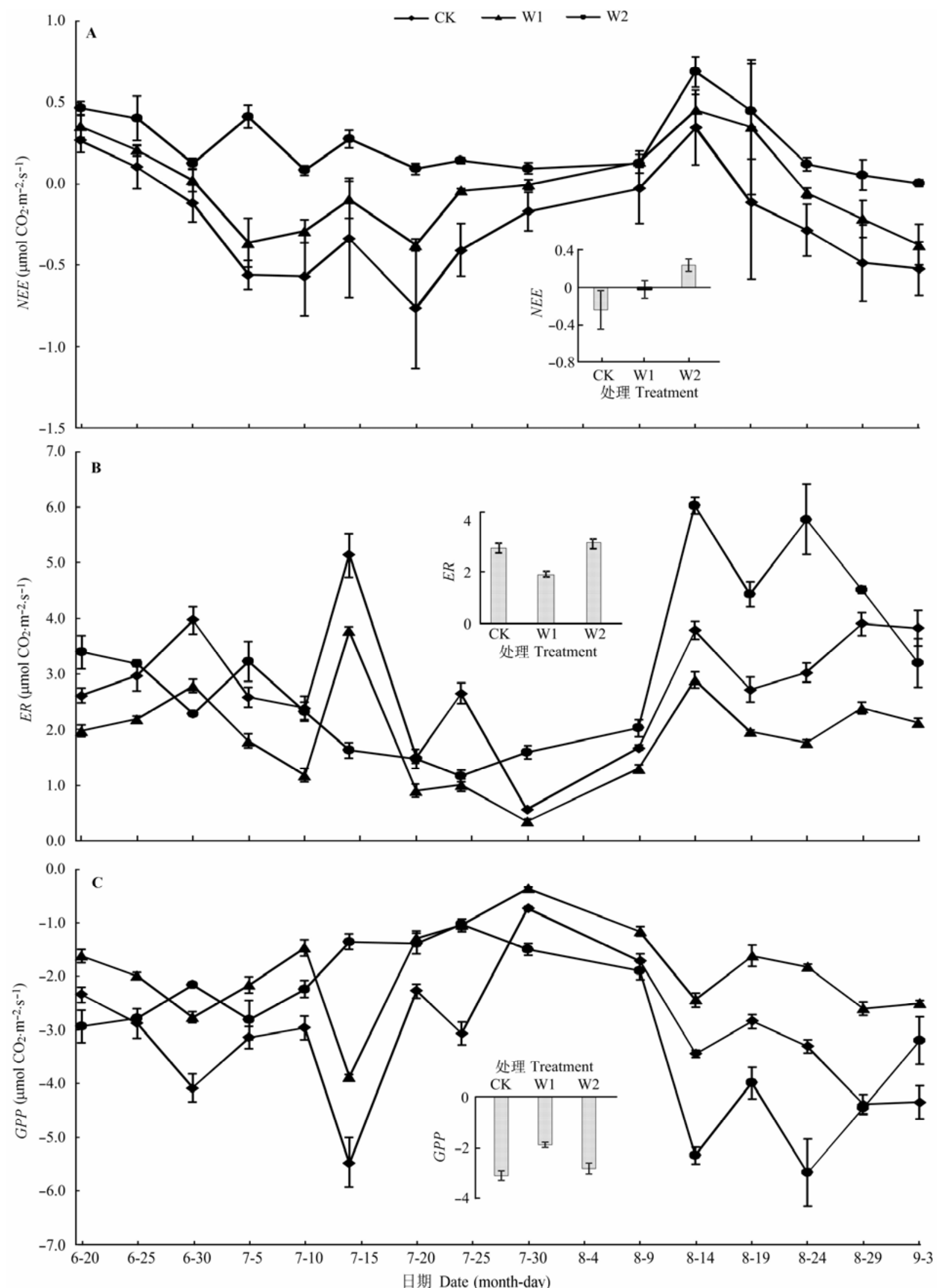

图3＼cjkstart植物生长季不同增温实验处理下的净生态系统碳交换(NEE, A)、生态系统呼吸 $(E R, \mathbf{B})$ 和生态系统总初级生产力 $(G P P, \mathbf{C})$ 的季节动态 (平均值土标准误差)。CK, 不增温; W1、W2分别代表模拟未来增温 $2{ }^{\circ} \mathrm{C}$ 和 $4{ }^{\circ} \mathrm{C}$ 的实验处理。

Fig. 3 Seasonal dynamics of net ecosystem $\mathrm{CO}_{2}$ exchange (NEE, A), ecosystem respiration (ER, B) and gross primary productivity $(G P P, \mathbf{C})$ during the growing season under different warming treatments (mean $\pm S E$ ). CK, no warming; W1, W2 represent the simulation of future temperature increase $2{ }^{\circ} \mathrm{C}$ and $4{ }^{\circ} \mathrm{C}$.

www.plant-ecology.com 

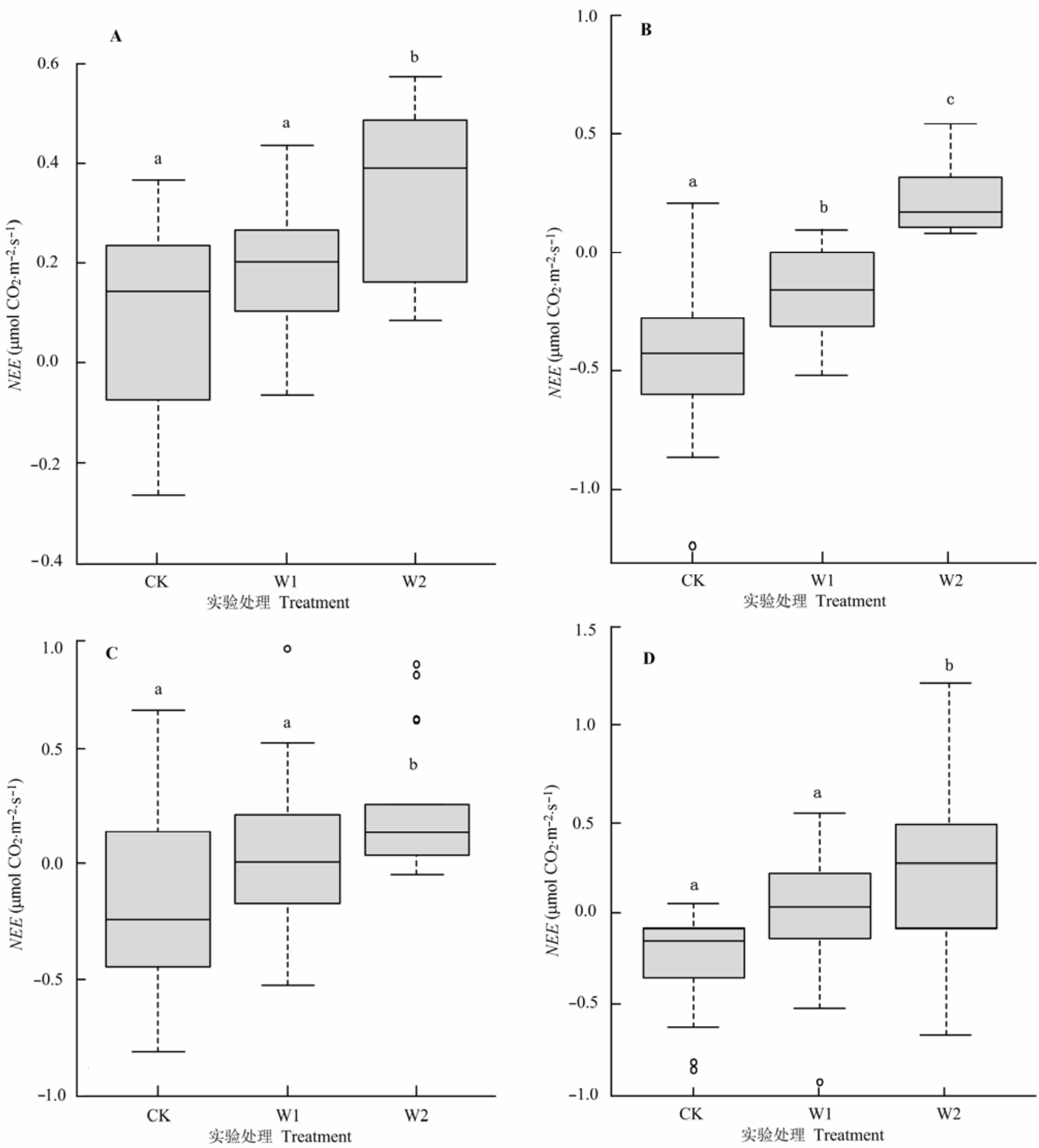

图4 植物生长季不同月份实验增温对净生态系统碳交换(NEE)的影响。A, 6月。 B, 7月。C, 8月。D, 2015年生长季。CK, 不 增温; W1、W2分别代表模拟未来增温 $2{ }^{\circ} \mathrm{C}$ 和 $4{ }^{\circ} \mathrm{C}$ 的实验处理。各图中上方的不同字母代表在 0.05 水平上差异显著。

Fig. 4 Different warming treatments effect on net ecosystem $\mathrm{CO}_{2}$ exchange (NEE) in different months during the growing season. A, June. B, July. C, August. D, Growing season in 2015. CK, no warming; W1, W2 represent the simulation of future temperature increase $2{ }^{\circ} \mathrm{C}$ and $4{ }^{\circ} \mathrm{C}$. The different letters at the top of the figures represent significant differences at the 0.05 level.

$\mathrm{CO}_{2} \cdot \mathrm{m}^{-2} \cdot \mathrm{s}^{-1}\left(p>0.05 ;\right.$ 图3C), 最终导致 $4{ }^{\circ} \mathrm{C}$ 增温表 现为碳源。

\section{$3.2 N E E$ 对增温响应的季节变异}

$N E E$ 是生态系统光合作用碳吸收与呼吸作用碳
排放的差值(张晴和李力, 2009), 土壤温湿度等环境 因子及地上生物量、叶面积指数等生物因子, 通过 直接影响生态系统的光合作用和呼吸作用而间接地 影响NEE (伏玉玲等, 2006; 黄祥忠等, 2006), 但由 


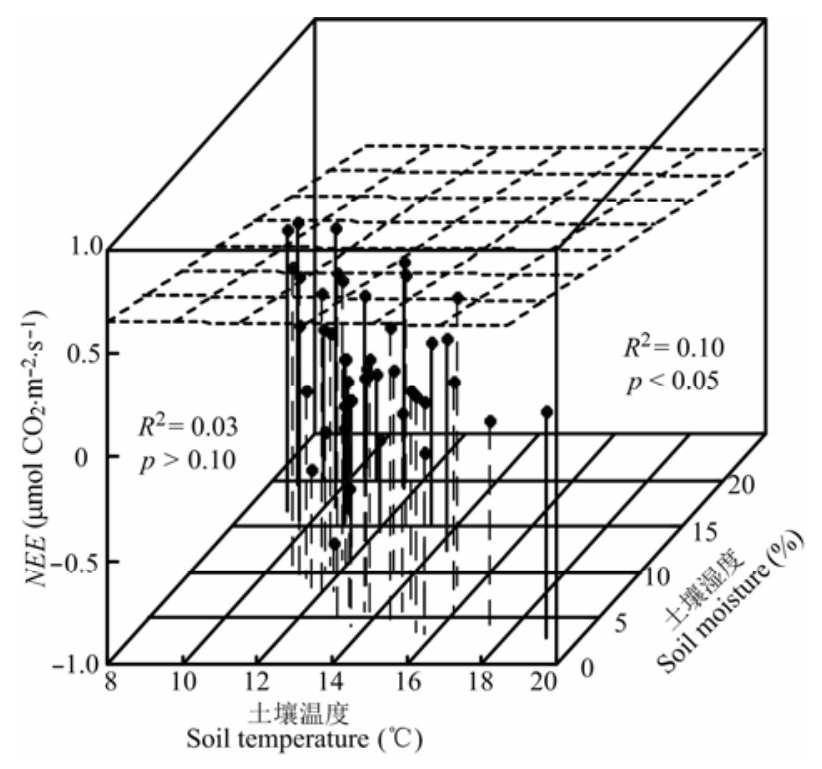

图5 土壤湿度、土壤温度与净生态系统碳交换(NEE)的回归 分析。

Fig. 5 Regression analysis for the relationships between net ecosystem $\mathrm{CO}_{2}$ exchange (NEE) and soil moisture, soil temperature.

于环境因子与生物因子在生长季的不同时间段差异 显著, 从而导致了N EE 对增温响应的季节差异。

我们的研究表明, 在生长季 6 月, 与不增温相 比, $4{ }^{\circ} \mathrm{C}$ 增温处理显著提高 $N E E$, 而 $2{ }^{\circ} \mathrm{C}$ 增温处理没 有显著改变 $N E E$; 不增温、 $2{ }^{\circ} \mathrm{C}$ 增温和 $4{ }^{\circ} \mathrm{C}$ 增温处理 下, 高寒草甸生态系统均表现为碳源。在藏北高寒 草甸生态系统, 6月植物群落中多数物种处于返青 期, 生态系统碳吸收较弱, ER 决定了 NEE, 而此时 降水较充足, 因此, 土壤温度成为植物生理活动的 限制性因素, 而增温可能提高ER (Kanerva et al., 2007; Strebel et al., 2010), 进而提高 $N E E$ 。因此, 在 生长季6月, 不增温、 $2{ }^{\circ} \mathrm{C}$ 增温和 $4{ }^{\circ} \mathrm{C}$ 增温处理均表 现为碳源, 与不增温相比, $4{ }^{\circ} \mathrm{C}$ 增温处理显著促进了 生态系统碳排放。

在7月, 与不增温相比, $2{ }^{\circ} \mathrm{C}$ 增温和 $4{ }^{\circ} \mathrm{C}$ 增温处 理均显著提高 $N E E$, 且 $2{ }^{\circ} \mathrm{C}$ 增温和 $4{ }^{\circ} \mathrm{C}$ 增温处理之 间差异显著; $4{ }^{\circ} \mathrm{C}$ 增温处理下, 高寒草甸生态系统 表现为碳源, 不增温和 $2{ }^{\circ} \mathrm{C}$ 增温处理表现为碳汇。相 比于温度和光照等环境因子, 降水的季节间与年际 间变化使降水成为影响各种生态系统生理、生态过 程的最普遍因素(Law et al., 2002; 伏玉玲等, 2006)。 在半干旱的高寒草甸生态系统中土壤水分是限制植 被生长及生态系统生产力的主要因素之一(Chen et al., 2015)。我们的研究也表明土壤水分是决定NEE
的关键因素(土壤湿度的相对重要性大于土壤温度 的相对重要性), 土壤含水量越高, 高寒草甸生态系 统碳吸收的能力越强, 干旱通过降低土壤水分进而 提高生态系统碳排放, 可能导致生态系统由碳汇变 为碳源(Meyers, 2001; Hunt et al., 2002), 并且增温 的负面效应在降水不足的情境下更为显著 (Dermody et al., 2007; Niu et al., 2008)。本研究中, 生长季7月降水量仅为 $40.1 \mathrm{~mm}, 4{ }^{\circ} \mathrm{C}$ 增温处理加剧 了土壤水分胁迫, 显著提高了 NEE, 在整个 7 月 4 ${ }^{\circ} \mathrm{C}$ 增温处理下, 高寒草甸生态系统表现为碳源。7月 降水主要集中在前期(7月1日-18日), 降水量为38.4 $\mathrm{mm}$, 后期(7月18日-30日)降水量为 $1.7 \mathrm{~mm}$, 因此, 在7月的前期, 不增温和 $2{ }^{\circ} \mathrm{C}$ 增温处理的碳吸收能力 逐渐增大, 而后期, 不增温和 $2{ }^{\circ} \mathrm{C}$ 增温处理的碳吸 收能力逐渐减小, 从整个7月来看, 不增温和 $2{ }^{\circ} \mathrm{C}$ 增 温处理下, 高寒草甸生态系统仍然表现为碳汇。

在8月, 与不增温相比, $4{ }^{\circ} \mathrm{C}$ 增温处理显著提高 $N E E$; 不增温处理下高寒草甸生态系统表现为碳汇, $2{ }^{\circ} \mathrm{C}$ 和 $4{ }^{\circ} \mathrm{C}$ 增温处理下高寒草甸生态系统均表现为 碳源。水分胁迫导致植物叶片气孔关闭、光合作用 和蒸腾作用降低, 植物生长缓慢(Baldocchi, 2003), 而不同根系深度的植物对增温导致的表层土壤水分 胁迫的响应和敏感性不同, 增温导致的浅层土壤水 分胁迫，对浅根植物的生长发育更为不利 (Porporato et al., 2001; Klein et al., 2008; Tsechor et al., 2013)。在藏北高寒草甸生态系统中, 浅根系的 高山嵩草是优势种, 占群落总盖度的60\%-85\% (朱 军涛, 2016), 在7月下旬, 高山嵩草受干旱胁迫严 重, 地上部分枯黄, 盖度降低(附件II)。Hao等(2008) 研究表明, 叶面积指数 ( $L A I)$ 是影响 NEE 的主要因 子, 与降水充足时期相比, 长期持续干旱使植物 $L A I$ 降低, 生态系统碳吸收能力降低。我们的研究数据 进一步支持上述结论, 在7月下旬, 由于受干旱胁迫 影响, 不增温、 $2{ }^{\circ} \mathrm{C}$ 增温和 $4{ }^{\circ} \mathrm{C}$ 增温条件下, 优势物 种高山嵩草盖度分别为 $24.4 \% 、 18.0 \%$ 和 $1.2 \%$ (附件 II), 因此导致从 8 月初到 8 月中旬, 所有处理的生态 系统碳排放逐渐增强, 且在8月中旬达到生长季的 最大值。8月中下旬随着降水量增加, 土壤水分得到 补充(图1), 所有处理的优势种高山嵩草的盖度都比 7月中旬提高, 分别为 $56.0 \% 、 55.0 \%$ 和 $20.0 \%$ (附件 II), 因此从 8 月中旬到 8 月末, 所有实验处理的生态 系统碳排放逐渐减弱, 在 8 月 20 日前后, 不增温和 
$2{ }^{\circ} \mathrm{C}$ 增温处理下, 高寒草甸生态系统由碳源转化为 碳汇, $4{ }^{\circ} \mathrm{C}$ 增温处理下仍表现为碳源。

\section{4 结论}

在全球变暖的背景下, 增温导致陆地生态系统 表现为碳源(Illeris et al., 2004)或碳汇(Niu et al., 2008)。我们的研究表明, 在半干旱的高寒草甸生态 系统, 土壤水分是决定 $N E E$ 的关键因素, 增温通过降 低土壤水分而导致高寒草甸生态系统碳汇能力下降, 增温促进了 NEE 的提高, 在未来 $2{ }^{\circ} \mathrm{C}$ 增温条件下, 高 寒草甸生态系统表现为弱碳汇, 而在 $4{ }^{\circ} \mathrm{C}$ 增温条件 下表现为强碳源。本研究预示着, 在未来更热、更干 的生长季, 青藏高原高寒草甸系统的碳吸收能力将 进一步下降。由于只有一个生长季的研究数据支持 该结论, 缺少实验结果的重复性验证, 今后将进行 重复观测和研究, 进一步完善上述研究结论。

基金项目 国家重点基础研究发展计划(973计划) (2013CB956302)、国家自然科学基金(41571195)和 中国科学院“西部之光”项目。

致谢 特别感谢中国科学院地理科学与资源研究所 博士研究生黄珂、组佳星等在西藏那曲野外观测工 作中给予的大力帮助。

\section{参考文献}

Baldocchi DD (2003). Assessing ecosystem carbon balance: Problems and prospects of the eddy covariance technique. Global Change Biology, 9, 478-492.

Chen J, Shi W, Cao J (2015). Effects of grazing on ecosystem $\mathrm{CO}_{2}$ exchange in a meadow grassland on the Tibetan Plateau during the growing season. Environmental Management, 55, 347-359.

Ciais PH, Reichstein M, Viovy N (2005). Europe-wide reduction in primary productivity caused by the heat and drought in 2003. Nature, 437, 529-533.

Corradi C, Kolle O, Walter K, Zimov SA, Schulze ED (2005). Carbon dioxide and methane exchange of a northeast Siberian tussock tundra. Global Change Biology, 11, 19101925.

Dermody O, Weltzin JF, Engel EC, Allen P, Norby RJ (2007). How do elevated $\left[\mathrm{CO}_{2}\right]$, warming, and reduced precipitation interact to affect soil moisture and $L A I$ in an old field ecosystem? Plant and Soil, 301, 255-266.

Fu BJ, Niu D, Zhao SD (2005). Global change and terrestrial ecosystems: Review and prospect. Earth Sciences, 20, 556-560. (in Chinese with English abstract) [傅伯杰, 牛 栋, 赵士洞 (2005). 全球变化与陆地生态系统研究: 回 顾与展望. 地球科学展, 20, 556-560.]
Fu YL, Yu GR, Wang YF, Li ZQ, Hao YB (2006). Effect of water stress on ecosystem photosynthesis and respiration of a Leymus chinensis steppe in Inner Mongolia. Science China, 36, 183-193. (in Chinese) [伏玉玲, 于贵瑞, 王艳 芬, 李正泉, 郝彦宾 (2006). 水分胁迫对内蒙古羊草草 原生态系统光合和呼吸作用的影响. 中国科学, 36, 183-193.]

Gao XQ (1994). Preliminary analysis of climate changes in some regions of north part of Qinghai-Xizang Plateau in decade year scale. In: Qinghai Tibet Project Expert Committee ed. Formation and Evolution, Environmental Changes and Ecosystem on the Qinghai-Xizang Plateau. Science Press, Beijing. 297-303. (in Chinese) [高晓清 (1994). 青藏高原 北部若干地点10年尺度气候变化的初步分析. 见: 青藏 项目专家委员会编. 青藏高原形成演化、环境变迁与生 态系统研究. 科学出版社, 北京. 297-303.]

Granier A, Reichstein M, Brédaa N, Janssens IA, Falge E, Ciais P, Grünwaldg T, Aubinet M, Berbigier P, Bernhofer C, Buchmann N, Facini O, Grassi G, Heinesch B, Ilvesniemi H, Keronen P, Knohl A, Köstner B, Lagergren F, Lindroth A, Longdoz B, Loustau D, Mateus J, Montagnani L, Nys C, Moors E, Papale D, Peiffer M, Pilegaard K, Pita G, Pumpanen J, Rambal S, Rebmann C, Rodrigues A, Seufert G, Tenhunen J, Vesala T, Wang Q (2007). Evidence for soil water control on carbon and water dynamics in European forests during the extremely dry year: 2003. Agricultural and Forest Meteorology, 143, 123-145.

Guo YQ, Borjigidai A, Gao QZ, Duan MJ, Ganzhu ZB, Wan YF, Li YE, Guo HB (2011). Photosynthetic characteristics of Stipa purpurea under irrigation in northern Tibet and its short-term response to temperature and $\mathrm{CO}_{2}$ concentration. Chinese Journal of Plant Ecology, 35, 311-321. (in Chinese with English abstract) [郭亚奇, 阿里穆斯, 高清竹, 段敏杰, 干珠扎布, 万运帆, 李玉娥, 郭红保 (2011). 灌溉条件下藏北紫花针茅光合特性及其对温度和 $\mathrm{CO}_{2}$ 浓度的短期响应. 植物生态学报, 35, 311-321.]

Hao YB, Wang YF, Mei XR, Huang XZ, Cui XY, Zhou XQ, Niu HS (2008). $\mathrm{CO}_{2}, \mathrm{H}_{2} \mathrm{O}$ and energy exchange of an Inner Mongolia steppe ecosystem during a dry and wet year. Acta Oecologia, 33, 133-143.

Harte J, Saleska S, Shih T (2006). Shifts in plant dominance control carbon-cycle responses to experimental warming and widespread drought. Environmental Research Letters, 1, 1-6.

Huang XZ, Hao YB, Wang YF (2006). Impact of extreme drought on net ecosystem exchange from Lemus chinensis steppe in Xilin River Basin, China. Chinese Journal of Plant Ecology, 30, 894-900. (in Chinese with English abstract) [黄祥忠, 郝彦宾, 王艳芬 (2006). 极端干旱条件 下锡林河流域羊草草原净生态系统碳交换特征. 植物 生态学报, 30, 894-900.] 
Hunt JE, Kelliher FM, McSeveny TM, Byers JN (2002). Evaporation and carbon dioxide exchange between the atmosphere and a tussock grassland during a summer drought. Agricultural and Forest Meteorology, 111, 65-82.

Illeris L, Christensen TR, Mastepanov M (2004). Moisture effects on temperature sensitivity of $\mathrm{CO}_{2}$ exchange in a subarctic heath ecosystem. Biogeochemistry, 70, 317-330.

IPCC (Intergovernmental Panel on Climate Change) (2007). Contribution of working group I to the fourth assessment report of the intergovernmental panel on climate change. In: Solomon S, Qin DH, Manning M, Marquis M, Chen ZL, Averyt K, Tignor M, Miller HL eds. Climate Change 2007: The Physical Science Basis. Cambridge University Press, Cambridge, UK.

Kanerva T, Regina K, Ramo K, Rämö K, Ojanperä K, Manninen $\mathrm{S}$ (2007). Fluxes of $\mathrm{N}_{2} \mathrm{O}, \mathrm{CH}_{4}$ and $\mathrm{CO}_{2}$ in a meadow ecosystem exposed to elevated ozone and carbon dioxide for three years. Environmental Pollution, 145, 818-828.

Klein JA, Harte J, Zhao XQ (2008). Decline in medicinal and forage species with warming is mediated by plant traits on the Tibetan Plateau. Ecosystems, 11, 775-789.

Law BE, Falge E, Gu L, Baldocchi DD, Bakwin P, Berbigier P, Davis K, Dolman AJ, Falk M, Fuentes JD, Goldstein A, Granier A, Grelle A, Hollinger D, Janssens IA, Jarvis P, Jensen NO, Katul G, Mahli Y, Matteucci G, Meyers T, Monson R, Munger W, Oechel W, Olson R, Pilegaard K, Paw KT, Thorgeirsson H, Valentini R, Verma S, Vesala T, Wilson K, Wofsy S (2002). Environmental controls over carbon dioxide and water vapor exchange of terrestrial vegetation. Agricultural and Forest Meteorology, 113, 97-120.

Li YN, Zhao L, Zhao XQ, Zhou HK (2004). Effects of a 5 -years mimic temperature increase to the structure and productivity of Kobresia humilis meadow. Acta Agrestia Sinica, 12, 236-239. (in Chinese with English abstract) [李 英年，赵亮，赵新全，周华坤 (2004). 5年模拟增温后矮 嵩草草甸群落结构及生产量的变化. 草地学报, 12, 236239.]

Liu X, Chen B (2000). Climatic warming in the Tibetan Plateau during recent decades. International Journal of Climatology, 14, 1729-1742.

Luo Y (2007). Terrestrial carbon-cycle feedback to climate warming. Annual Review of Ecology Evolution and Systematics, 38, 683-712.

Melillo JM, Steudler PA, Aber JD, Newkrik K, Lux H, Bowles FP, Catricala C, Magill A, Ahrens T, Morrisseau S (2002). Soil warming and carbon-cycle feedbacks to the climate system. Science, 298, 2173-2175.

Meyers TP (2001). A comparison of summertime water and $\mathrm{CO}_{2}$ fluxes over rangeland for well watered and drought conditions. Agricultural and Forest Meteorology, 106,
205-214.

Niu SL, Wu MY, Han Y, Xia JY, Li MH, Wan SQ (2008). Water-mediated responses of ecosystem carbon fluxes to climatic change in a temperate steppe. New Phytologist, 177, 209-219.

Niu S, Sherry R, Zhou X, Luo Y (2013). Ecosystem carbon fluxes in response to warming and clipping in a tall grass prairie. Ecosystems, 6, 948-961.

Oberbauer SF, Tweedie CE, Welker JM, Fahnestock JT, Henry GHR, Webber PJ, Hollister RD, Walker MD, Kuchy A, Elmore E, Starr G (2007). Tundra $\mathrm{CO}_{2}$ fluxes in response to experimental warming across latitudinal and moisture gradients. Ecological Monographs, 77, 221-238.

Peñuelas J, Gordon C, Llorens L, Nielsen T, Tietema A, Beier C, Bruna P, Emmett B, Estiarte M, Gorissenet A (2004). Non-intrusive field experiments show different plant responses to warming and drought among sites, seasons and species in a North-South European gradient. Ecosystems, 7, 598-612.

Porporato A, Laio F, Ridolfi L, Rodriguez-Iturbe I (2001). Plants in water-controlled ecosystems: Active role in hydrologic processes and response to water stress: III. Vegetation water stress. Advances in Water Resources, 24, 725-744.

Qi WW, Niu HS, Wang SP, Liu YJ, Zhang LR (2012). Simulation of effects of warming on carbon budget in alpine meadow ecosystem on the Tibetan Plateau. Acta Ecologica Sinica, 30, 1713-1722. (in Chinese with English abstract) [元伟伟, 牛海山, 汪诗平, 刘艳杰, 张立荣 (2012). 增 温对青藏高原高寒草甸生态系统固碳通量影响的模拟 研究. 生态学报, 30, 1713-1722.]

Rustad LE, Campbell JL, Marion GM, Norby RJ, Mitchell MJ, Hartley AE, Cornelissen JHC, Gurevitch J (2001). A meta-analysis of the response of soil respiration, net nitrogen mineralization, and aboveground plant growth to experimental ecosystem warming. Oecologia, 14, 543-562.

Saleska SR, Harte J, Torn MS (1999). The effect of experimental ecosystem warming on $\mathrm{CO}_{2}$ fluxes in a montane meadow. Global Change Biology, 5, 125-141.

Strebel D, Elberling B, Morgner E, Knicker HE, Cooper EJ (2010). Cold-season soil respiration in response to grazing and warming in High Arctic Svalbard. Polar Research, 29, 46-57.

Thomas CD, Cameron A, Green RE, Bakkenes M, Beaumont LJ, Collingham YC, Erasmus BF, de Siqueira MF, Grainger A, Hannah L, Hughes L, Huntley B, van Jaarsveld AS, Midgley GF, Miles L, Ortega-Huerta MA, Peterson AT, Phillips OL, Williams SE (2004). Extinction risk from climate change. Nature, 427, 145-148.

Tsechor DJ, Totland O, Moe SR, Hopping KA, Pan JB, Klein JA (2013). Plant functional traits mediate reproductive phenology and success in response to experimental 
warming and snow addition in Tibet. Global Change Biology, 19, 459-472.

Wan SQ, Hui D, Wallace LL, Luo Y (2005). Direct and indirect warming effects on ecosystem carbon processes in a tall grass prairie. Global Biogeochemical Cycles, 19, 1-13.

Wan SQ, Norby RJ, Ledford J, Weltzin JF (2007). Responses of soil respiration to elevated $\mathrm{CO}_{2}$, air warming, and changing soil water availability in a model old-field grassland. Global Change Biology, 13, 2411-2424.

Welker JM, Fahnestock JT, Henry GR, O’Dea KW, Chimner $\mathrm{RA}$ (2004). $\mathrm{CO}_{2}$ exchange in three Canadian high Arctic ecosystems: Response to long-term experimental warming. Global Change Biology, 10, 1981-1995.

Xia JY, Niu SL, Wan SQ (2009). Response of ecosystem carbon exchange to warming and nitrogen addition during two hydrologically contrasting growing seasons in a temperate steppe. Global Change Biology, 15, 1544-1556.

Zhang JX, Cao GM, Zhou DW, Hu QW, Zhao XQ (2003). Alpine meadow air-soil-vegetation-animal system carbon storage and carbon cycle. Acta Ecologica Sinica, 23, 627-634. (in Chinese with English abstract) [张金霞, 曹 广民, 周党卫, 胡启武, 赵新全 (2003). 高寒矮嵩草草

附件I 增温处理实验设计。

Appendix I The experimental design of warming treatment.

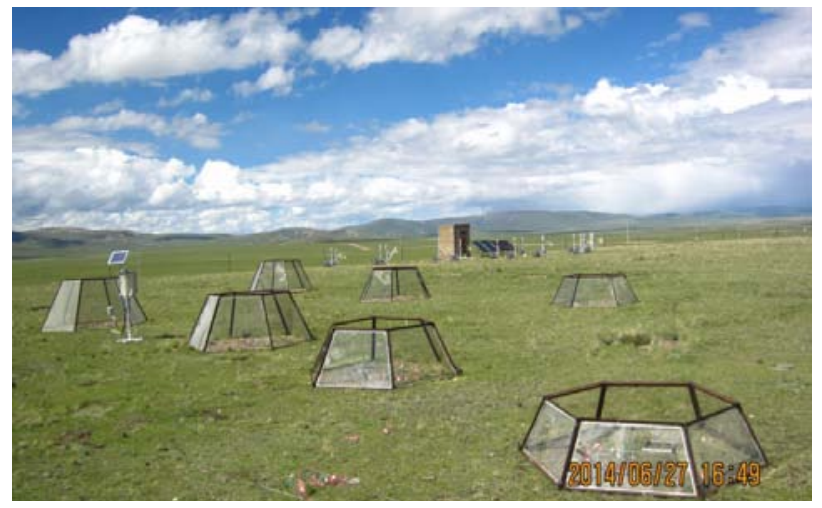

甸大气-土壤-植被-动物系统碳素储量及碳素循环. 生 态学报, 23, 627-634.]

Zhang Q, Li L (2009). Study on the spatial-temporal change characteristics of net ecosystem exchange (NEE) in China. Journal of Anhui Agricultural Sciences, 37, 3108-3109. (in Chinese with English abstract) [张晴, 李力 (2009). 我国净生态系统碳交换量(NEE)的时空变化特征研究. 安徽农业科学, 37, 3108-3109.]

Zhou XH, Wan SQ, Luo YQ (2007). Source components and interannual variability of soil $\mathrm{CO}_{2}$ efflux under experimental warming and clipping in a grassland ecosystem. Global Change Biology, 13, 761-775.

Zhu JT (2016). Effects of experimental warming on plant reproductive phenology in Xizang alpine meadow. Chinese Journal of Plant Ecology, 40, 1028-1036. (in Chinese with English abstract) [朱军涛 (2016). 实验增温对藏北高寒 草甸植物繁殖物候的影响. 植物生态学报, 40, 10281036.]

责任编委: 孙书存 责任编辑: 王 葳

附件II 2015年生长季高山嵩草盖度。CK, 不增温, W1、 W2分别代表模拟未来增温 $2{ }^{\circ} \mathrm{C}$ 和 $4{ }^{\circ} \mathrm{C}$ 的实验处理。

Appendix II The coverage of Kobresia pygmaea during growing season in 2015. CK, no warming; W1, W2 represent the simulation of future temperature increase $2{ }^{\circ} \mathrm{C}$ and $4{ }^{\circ} \mathrm{C}$.

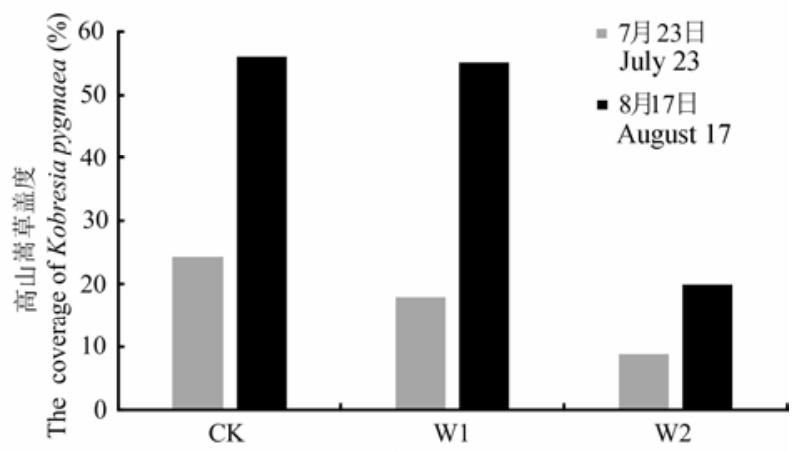

实验处理 Treatment

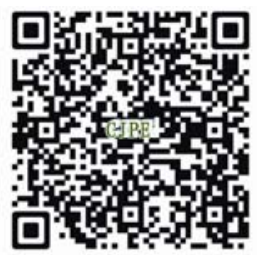

植物生态学报官网

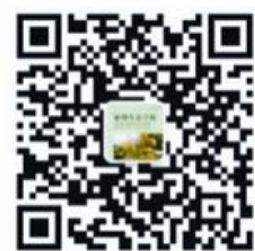

微信订阅号

期刊及学科

相关信息发布

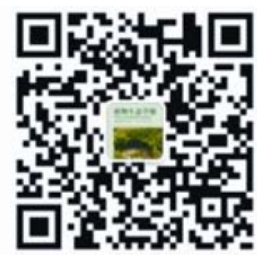

微信服务号

稿件状态查询

全文检索汶览 